\title{
A new kind of auxiliary heart in insects: functional morphology and neuronal control of the accessory pulsatile organs of the cricket ovipositor
}

\author{
Reinhold Hustert ${ }^{1}$, Matthias Frisch ${ }^{1}$, Alexander Böhm² and Günther Pass ${ }^{2 *}$
}

\begin{abstract}
Introduction: In insects, the pumping of the dorsal heart causes circulation of hemolymph throughout the central body cavity, but not within the interior of long body appendages. Hemolymph exchange in these dead-end structures is accomplished by special flow-guiding structures and/or autonomous pulsatile organs ("auxiliary hearts"). In this paper accessory pulsatile organs for an insect ovipositor are described for the first time. We studied these organs in females of the cricket Acheta domesticus by analyzing their functional morphology, neuroanatomy and physiological control.
\end{abstract}

Results: The lumen of the four long ovipositor valves is subdivided by longitudinal septa of connective tissue into efferent and afferent hemolymph sinuses which are confluent distally. The countercurrent flow in these sinuses is effected by pulsatile organs which are located at the bases of the ovipositor valves. Each of the four organs consists of a pumping chamber which is compressed by rhythmically contracting muscles. The morphology of the paired organs is laterally mirrored, and there are differences in some details between the dorsal and ventral organs. The compression of the pumping chambers of each valve pair occurs with a left-right alternating rhythm with a frequency of 0.2 to $0.5 \mathrm{~Hz}$ and is synchronized between the dorsal and ventral organs. The more anteriorly located genital chamber shows rhythmical lateral movements simultaneous to those of the ovipositor pulsatile organs and probably supports the hemolymph exchange in the abdominal apex region. The left-right alternating rhythm is produced by a central pattern generator located in the terminal ganglion. It requires no sensory feedback for its output since it persists in the completely isolated ganglion. Rhythm-modulating and rhythm-resetting interneurons are identified in the terminal ganglion.

Conclusion: The circulatory organs of the cricket ovipositor have a unique functional morphology. The pumping apparatus at the base of each ovipositor valve operates like a bellow. It forces hemolymph via sinuses delimited by thin septa of connective tissue in a countercurrent flow through the valve lumen. The pumping activity is based on neurogenic control by a central pattern generator in the terminal ganglion.

Keywords: Orthoptera, Gryllidae, Abdomen, Circulation, Hemolymph, Neuroanatomy, Neurogenic, Terminal ganglion, Central pattern generator, Evolutionary novelty

\section{Introduction}

In the open circulatory system of insects, the pumping dorsal heart tube circulates hemolymph in the central body cavity enabling a constant perfusion of the internal organs and tissues. This flow, however, cannot effect circulation in outlying dead-end structures, such as antennae, legs, wings and abdominal appendages. For this

\footnotetext{
* Correspondence: guenther.pass@univie.ac.at

${ }^{2}$ Department of Integrative Zoology, University of Vienna, Althanstraße 14, 1090 Vienna, Austria

Full list of author information is available at the end of the article
}

task, insects have special hemolymph guiding structures and/or auxiliary hearts [1-3].

In appendages, such as the thoracic legs and some abdominal appendages, a longitudinal septum divides the lumen into two sinuses. Distally the septum is lacking, and the sinuses are confluent. Thereby a countercurrent flow is enabled within these appendages, and we distinguish between an efferent and afferent sinus. How the hemolymph flow is produced remains unclear in most cases $[3,4]$. In some appendages, pressure changes due to regular volume alterations of tracheae or tracheal 
sacs contribute to the hemolymph exchange [5,6]. More elaborate organs for the supply of hemolymph to long body appendages are the so-called accessory pulsatile organs or auxiliary hearts. These muscle-driven pumps can be very diverse in their functional morphology in the various groups of insects. They may be located at the base or within the appendages and are in general autonomous organs which pump rhythmically, but independently, from that of the dorsal heart. The contractions of these auxiliary hearts are based on a myogenic automatism which can be modulated by neuronal and/or neurohormonal control [7-10]. A thoroughly investigated example of such an auxiliary heart is the antenna-heart of the cockroach Periplaneta americana in which the functional morphology, neuroanatomy, neurochemistry, pharmacology and the control mechanisms have been analyzed in detail [11-16].

However, the problem of circulation has not yet been investigated in insect ovipositors although some of them reach considerable length. In this paper we describe for the first time accessory pulsatile organs for these body appendages. The organs were discovered in the female cricket Acheta domesticus (preliminary notes [1,17]). In live specimens, hemocyte movements can be observed under the microscope through transparent parts of the ovipositor cuticle. The flow occurs in pulses that are clearly correlated with conspicuous compressions of structures at the base of the ovipositor valves which were revealed to be the pumping organs for hemolymph circulation in these appendages. The functional morphology of these ovipositor pulsatile organs was investigated on the basis of serial semi-thin sections and a microCT scan in combination with in vivo observations. In addition, neuroanatomical and physiological studies were performed. Several motoneurons and interneurons involved in the control of the ovipositor pulsatile organs could be identified in the terminal ganglion. The electrophysiological recordings revealed a coordinated and rhythmic bilateral motor output from these neurons. Since the rhythm persists even when the terminal ganglion is completely isolated, it could serve as a model for studies of autonomous rhythm generation in a neural network (preliminary reports $[17,18])$.

\section{Results}

Ovipositor and anatomical condition at the abdominal apex

The ovipositor shaft is composed of four long valves: a ventral pair, referred to as the gonapophyses of the abdominal segment 8 (ga8; synonyms: $1^{\text {st }}$ valvulae of Snodgrass $[19,20], 1^{\text {st }}$ gonapophyses of Scudder [21]), and a dorsal pair, referred to as the lateral gonapophyses of the abdominal segment 9 (ga9l; $3^{\text {rd }}$ valvulae of Snodgrass $[19,20]$, gonoplacs of Scudder [21]). The median gonapophyses of the $9^{\text {th }}$ segment (ga9m, synonyms: $2^{\text {nd }}$ valvulae of Snodgrass $[19,20], 2^{\text {nd }}$ gonapophyses of Scudder [21]) are very short and inconspicuous structures in crickets. The long ovipositor valves are distinctly widened at their bases and interconnected by several joints to the highly modified and strongly sclerotized coxosternites of the abdominal segment 8 and 9, respectively (cs8 and cs9; synonyms: $1^{\text {st }}$ and $2^{\text {nd }}$ valvifers of Snodgrass $[19,20]$, gonocoxae of Scudder [21], coxosternite of Klass and Ulbricht [22]). The lumen of the four long ovipositor valves is subdivided by delicate septa of connective tissue into sinuses in which efferent or afferent flows of hemolymph can be observed in live specimens. Aside from the epidermis, the ovipositor valves only contain nerves and few tracheal trunks extending to the apical tip. The ovipositor pulsatile organs (opo) are located at the base of the appendages (Figure 1).

\section{Ovipositor pulsatile organs of the abdominal segment $\mathbf{8}$}

Removal of the subgenital plate exposes the bases of the ga8 (Figure 1A, B; Figure 2A). Their lateral sides consist of a strongly sclerotized cuticle while medially the cuticle is thin and flexible forming a conspicuous bulge at each ga8 base ("soft lateral walls" of [23]). The two bulges form the soft-walled margin of the genital opening. In live specimens conspicuous movements of the bulges can be observed: they are strongly compressed and expanded in an alternating left-right rhythm of $0.2-0.5 \mathrm{~Hz}$ at room temperature (see Additional file 1: Video). Our study concludes that the compressible bulges constitute the pumping chambers of the ovipositor pulsatile organs of abdominal segment 8 (opo8). They serve as bellow-like pumping devices for hemolymph transport through the ga8. The compression is caused by a muscle that extends across the lumen of the bulges ( $\mathrm{cm} 8$, Figure $2 \mathrm{~B}$ ). The muscle originates laterally at the sclerotized cs8 and fans out into individual strands which attach medially to the soft wall of the bulge (Figures 3A, B; Figure 4D). Contraction of this muscle pulls the soft parts of the cuticle in a lateral direction. Thereby the pumping chamber is compressed, and hemolymph is forced into the ga8. The individual muscle strands are extended at slightly different angles enabling compression of a wide area of the bulge during contraction. The compression of one bulge supports the dilation of the other bulge and thereby helps to fill it with hemolymph drawn from the abdominal body cavity. In this way the two opo8 operate as two partly interconnected left-right alternating bellows.

After each pumping stroke the hemolymph slightly flows back before it stalls. This observation is considered an indication for the absence of a discrete valve device within the ga8. However, on each body side some of the more anterior and dorsal muscle strands of the $\mathrm{cm} 8$ are attached to the lateral margins of the flat cuticle part between the two bulges (Figure 3A, arrow). During bulge 

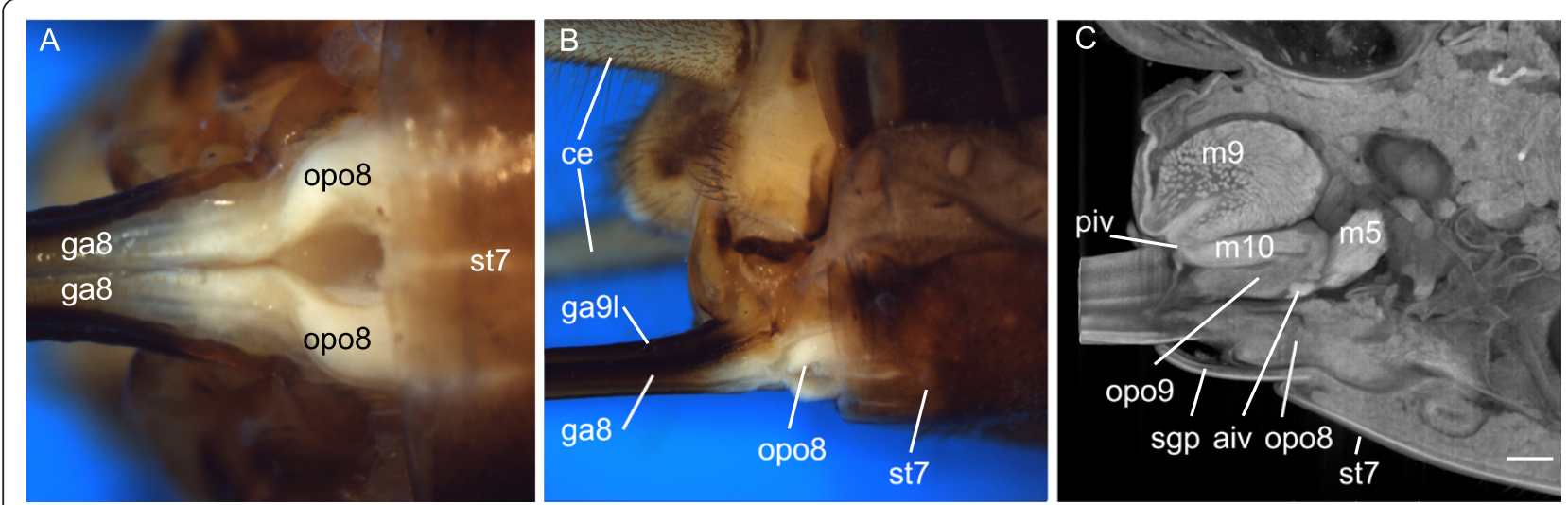

Figure 1 Location of the ovipositor pulsatile organs in the abdominal apex. (A) Ventral view (subgenital plate removed): the distally strongly sclerotized paired gonapophyses 8 (ga8) have proximally an extensive area of thin and flexible cuticle. The distinct bulges at their basal articulation represent the ovipositor pulsatile organs 8 (opo8). (B) Lateral view (subgenital plate removed): the ovipositor shaft consists of the ga8 and the lateral gonapophysis 9 (ga9l); opo8 form protruding bulges. (C) Volume rendering of microCT data in lateral view showing spatial relationship of opo8 and opo9 to the anterior and posterior intervalvular sclerites (aiv, piv) and some larger muscles (numbering follows [19]). Scale bar: $500 \mu \mathrm{m}$. Further abbreviations: ce, cercus; sgp, subgenital plate; st7, sternite of the $7^{\text {th }}$ abdominal segment.

compression this part is pulled strongly into a lateral direction thereby narrowing the base of the bulge. This movement may partly prevent backflow of hemolymph into the abdominal cavity.

The lumen of each bulge is divided by a septum into a wide median sinus ( $\mathrm{si8m}$ ) which contains the $\mathrm{cm} 8$ in its proximal portion, and a narrower lateral sinus (si8l) which is devoid of any muscles (Figure 3A; Figure 4C). The septum extends into the ga8 nearly up to the apex and separates the somewhat smaller si8m from the larger si81 (Figure 5A, B). Compressions of the opo8 force hemolymph into a distal direction within the si8m. Observed peak flow velocities of hemocytes range between 5 to $15 \mathrm{~mm} \cdot \mathrm{s}^{-1}$, and hemolymph can thereby be moved from the base to the apex of the ga8 with a single pumping stroke. At the lancet-like apex of the ga8, the septum is perforated and the hemolymph can flow into the lateral sinus. There it returns to the base of the ga8 and continues further back dorso-laterally into the body cavity.

Two tracheae are embedded within the septum which separates the si8m and si8l in each ga8 (Figure 4C, D). During each pumping stroke these tracheae become laterally displaced and partly compressed. When the ipsilateral bulge relaxes, the tracheae return to their original shape indicating that during pumping strokes the hemolymph pressure in the si8m is higher than in the si8l.

\section{Ovipositor pulsatile organs of the abdominal segment 9}

The pump system of the ovipositor pulsatile organs 9 (opo9) overlies the posterior part of the opo8 (Figure 2C, D). Its functional principle resembles that of the abdominal segment 8 , but there are some anatomical differences which may be explained by the deeper integration of the ga9 bases into the abdominal apex. The soft cuticular parts of the opo9 (Figure 4C, D) extend ventrally between the lateral strongly sclerotized parts of the coxosternite 9 (cs9), as well as the anterior and posterior intervalvular sclerites (aiv and piv after Snodgrass [19], Figure 2D). Medially, the walls of the two ga9 bases are narrowly apposed which appears from outside as slit-like invagination (Figure 4A, B). It consists for the most part of flexible cuticle but in the midline, where the right and left ga9 meet, there is a strongly sclerotized structure (Figure 3A). The small hemocoel spaces lateral of the invagination represent the pump chambers of opo9. Each chamber is continuous with the ventral sinus (si9v) of the ipsilateral ga9 (Figures 2A, 3A, 4 and 5), and dorsally each chamber is covered by a muscle $(\mathrm{cm} 9)$ that is attached to the upper part of the invagination and laterally to the cs9 (Figure 3A; Figure 4C, D). In live specimens one can observe that alternating contractions of the left and right muscles tilt the median cuticular structure and the flexible median cuticle portion to the corresponding side (see Additional file 1: Video). Thereby the two pump chambers are compressed and widened in alternation and hemolymph is forced into the si9v of each ga9l. The opo9 operates, similar to the opo8, as a pair of interconnected left-right alternating bellows: compression of one pump chamber (systole) simultaneously widens the opposite chamber (diastole) and stretches its compressor muscles ( $\mathrm{cm} 9)$. This leads to contraction of the $\mathrm{cm} 9$ thereby completing a full pumping cycle.

Hemolymph flows through the si9v of ga9l to the apex and passes through small gaps in the septa to si9d and si9i (Figure 5C, D). From these two sinuses the hemolymph flows back to the ovipositor base. During each pumping 

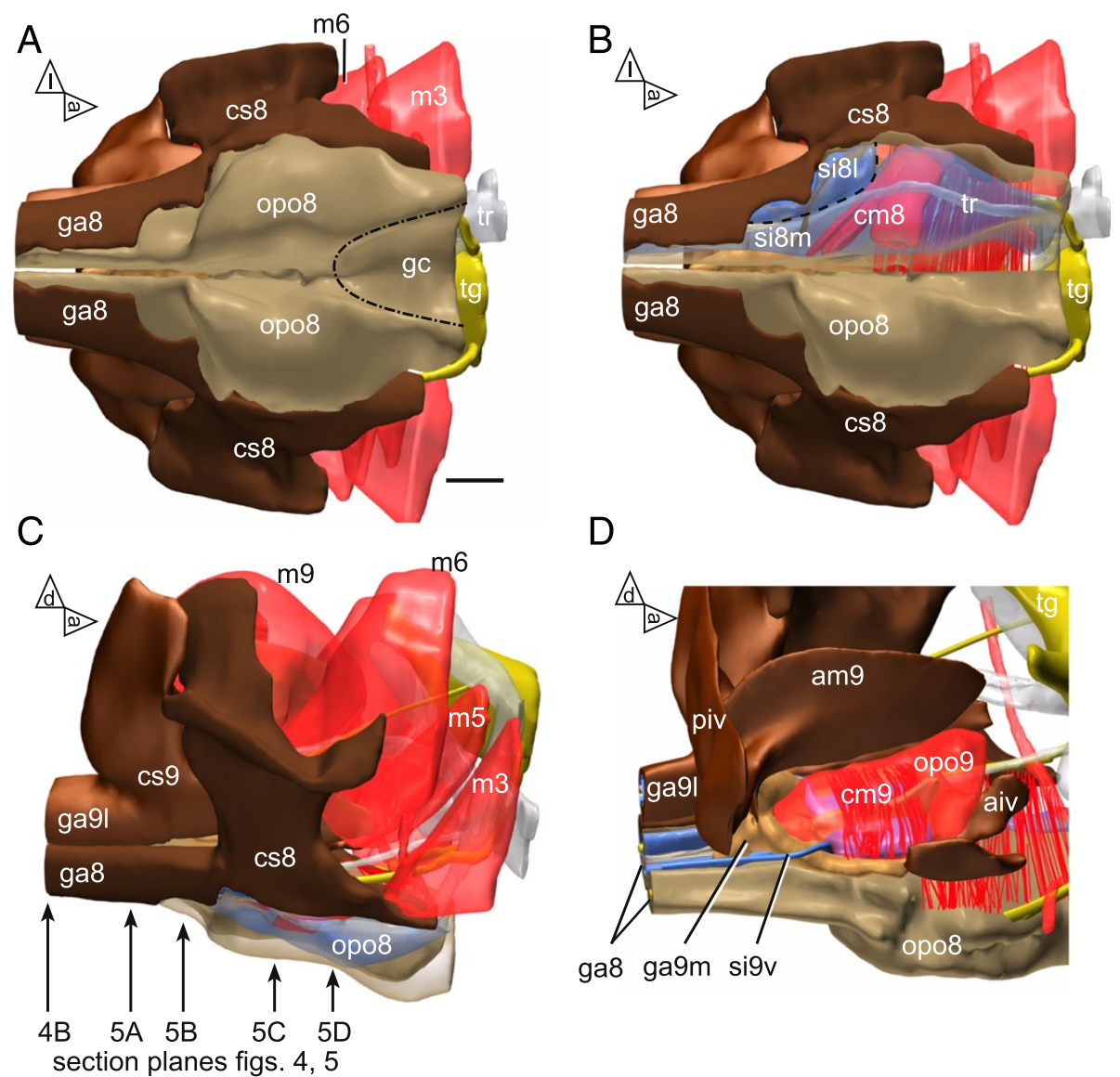

Figure 2 Reconstruction of the ovipositor pulsatile organs and the surrounding structures of the abdominal apex. (A) Ventral view (subgenital plate). Bases of the gonapophyses 8 (ga8) enlarged and with strongly sclerotized lateral parts (dark brown), medial parts of flexible cuticle (light brown) forming compressible bulges which constitute the ovipositor pulsatile organ 8 (opo8); area of the preceding genital chamber (gc) delimited by dotted line. (B) Same view as in A, but right ga8 base is presented transparently; compressor muscle 8 (cm8) (red) extends between strongly sclerotized coxosternite 8 (cs8) and medial flexible cuticle of ga8; lumen of ga8 is divided by a delicate septum (indicated by dashed line) into a wide medial sinus (si8m) and a smaller lateral sinus (si8l; both sinuses in blue) (C) Lateral view. ga8 and lateral gonapophysis 9 (ga9l) are articulately jointed to cs8 and cs9. The bulges of the protruding ovipositor pulsatile organ (opo8) are presented transparently. Arrows refer to section levels of Figures 4 and 5. (D) Parasagittal view of the opo, most of left cs9 and many large muscles are removed to expose opo9; cm9 extends between cs 9 and a median invagination of flexible cuticle. Scale bar: $250 \mu \mathrm{m}$. Further abbreviations (numbers 8 and 9 refer to the respective abdominal segment): aiv, anterior intervalvular sclerite; am, median apodeme (attachment site of muscle 9); m, muscle (numbering follows [19]); piv, posterior intervalvular sclerite; tr, trachea; tg, terminal ganglion. Coloring of structures in italics. Arrowheads outside the diagram indicate reference planes: $a$, anterior; $d$, dorsal; l, lateral.

stroke the tracheae in the si9d of the ga9l (Figure 4B) become displaced and partly collapse; in the intervals they return to their original position.

\section{Genital chamber movements}

The regular pumping of the left and right opos is well synchronized with a rhythmic bilateral movement of the genital chamber (gc) located in the posterior ventral abdomen (Figure 6). It is caused by the attached bilateral muscles (m2 of Snodgrass [19]) which originate laterally at the sternite of the abdominal segment 7 . The tilting of the gc narrows and widens the lateral hemolymph spaces. In intact animals these movements are clearly visible through the transparent parts of the abdominal sternite 7 and were the first and most obvious indication for the presence of the rhythmic pumping apparatus at the base of the ovipositor valves in Acheta domesticus [24].

The gc muscles $(\mathrm{m} 2)$ are always active in synchrony with the ipsilateral opo8/9 contraction muscles as was evident from long-time recording in more than 25 preparations. However, they can halt or remain in tonic contraction when they contribute to other behavior; the opo8/9 muscles however continue their rhythm at the same time.

\section{Innervation and rhythm of the accessory hearts}

The terminal ganglion (tg) is a fusion of the abdominal ganglia of the $7^{\text {th }}$ and the more posterior abdominal 


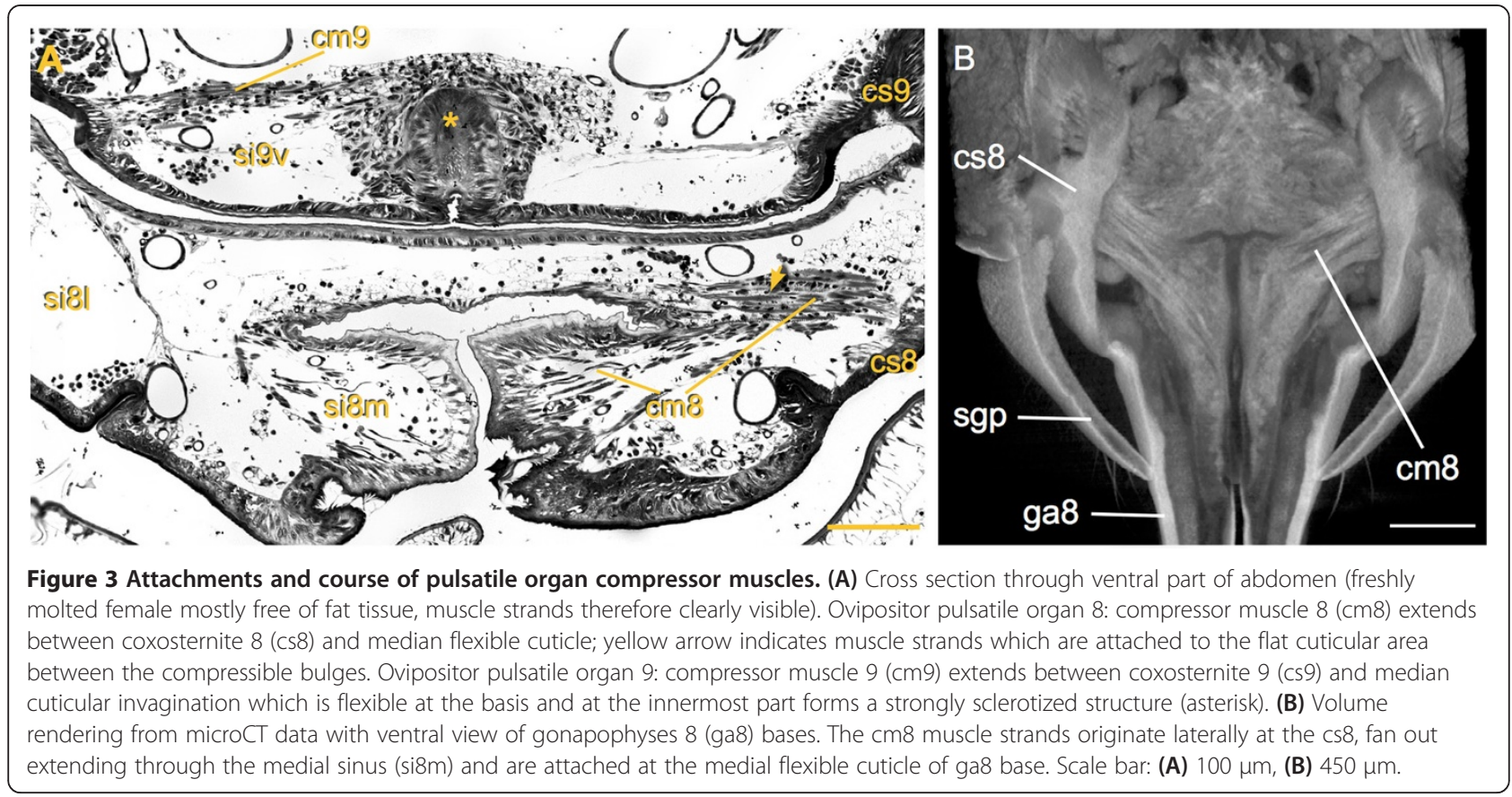

segments. Its ventral nerves innervate the sternal regions of several segments $(7 v, 8 v, 9 v)$ including the motor innervation of the rhythmic pumping muscles of the opos and the gc. Experiments in which the specific nerve branches that innervate the pumping muscle of an opo are cut selectively show that the contraction is based on neural commands. After denervation it stops immediately and permanently. The pumping muscles of the other opos remain active during these experiments as long as their nerve connection to the tg persists.

The rhythmic motor output recorded from the tilting muscles of the gc or the opos typically shows the activity of two excitatory motor units bursting in phase. One unit usually bursts at a higher frequency and longer than the other (Figure 7B, C). The somata of the rhythmic motoneurons for the opos are located ipsilaterally in their respective neuromere. Their neurites exhibit a rich branching, also into the neighboring neuromeres and some even with extensions across the midline (Figure 7B, C).

The opo rhythms of intact animals are occasionally accelerated or decreased respectively terminated during certain activities of the abdomen such as oviposition or strong ventilation. Our intracellular recordings from opo motoneurons reveal that the input, which elicits rhythmic motor bursts, starts with distinct excitatory postsynaptic potentials from premotor interneurons (in) which summate and release efferent spikes when the membrane potential rises over the firing threshold of the motoneuron (Figure 7B, C).

\section{Influences on pattern generation for opos in the} terminal ganglion

The pattern generation for opo pumping originates from the tg. While in intact and freely moving animals the motor output to the pumping muscles can be variable, the auxiliary tilting of the gc by the $\mathrm{m} 2$ remains consistently regular after severing the neck connectives and persists for several hours (Figure 7A). The common rhythm of the central pattern generator (cpg) even persists after severance of all peripheral nerves and the anterior connectives of the tg. The cpg thereby becomes separated from all sensory inputs and signals from the other ganglia of the cns. In such an isolated tg, the rhythms remain regular at about $0.5-0.2 \mathrm{~Hz}$ in aerated saline. Using this preparation we could also substantiate several non-neural influences on the autonomous rhythm generation and bilateral coordination of the cpg in the tg:

(i) Temperature changes applied unilaterally to the tg (Figure 8). By bringing warm or cold Peltier probes close to one side, changes in the left-right alternating rhythm of the opos were provoked. Dramatic inhibitory effects on the rhythm are caused by cooling, which when applied unilaterally decreases and abolishes the ipsilateral motor output of the cpg while the contralateral pattern paces down to a slower rhythm with extended burst durations. Removal of the cold probe gradually restores the initial bilateral rhythm.

(ii) Different concentrations of $\mathrm{CO}_{2}$. Infusion of air with gradually increasing $\mathrm{pCO}_{2}$ into the lateral trachea that supplies an isolated tg (Figure 9A) slows the ipsilateral rhythmic motor output to the opos progressively and the 


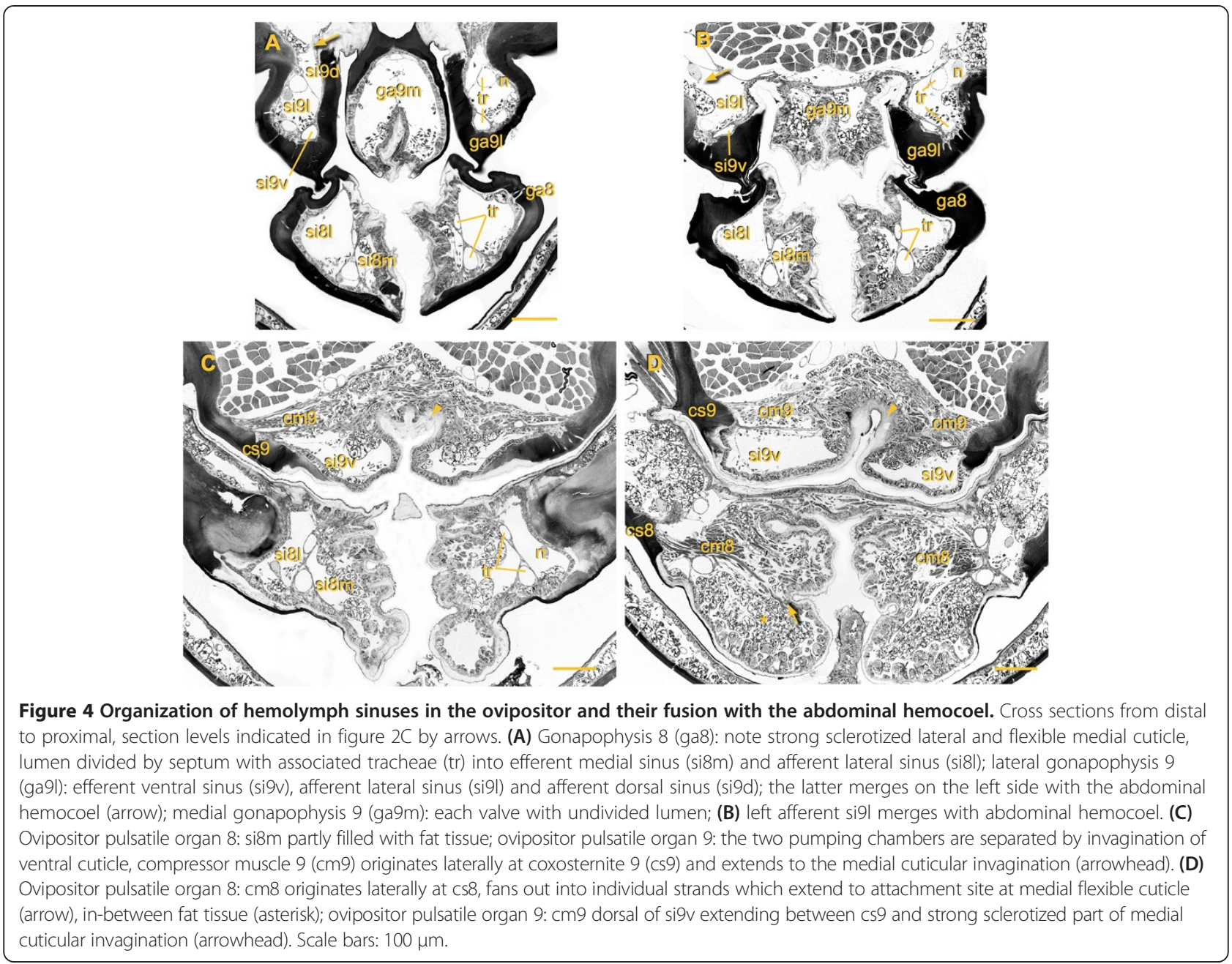

amplitudes of action potentials decrease (Figure 9B). Finally the rhythm ceases in one hemiganglion while the regular rhythm of the other side persists. Stopping the $\mathrm{CO}_{2}$ infusion allows for the rhythm to recover and return to the initial rates. In contrast, when the ganglion surface is superfused with bathing saline in which the $\mathrm{pCO}_{2}$ is increased (which also lowers the $\mathrm{pH}$ of the saline), the cpg rhythm accelerates and finally transits into more tonic activity (Figure 9C).

(iii) Increasing acidity (with drops of $\mathrm{HCl}$ ) of the saline bathing of the ganglion. This procedure had an accelerating effect on the cpg for the opo rhythms from 0.23 to $0.26 \mathrm{~Hz}$ and also lowered the action potential amplitudes (Figure 9D). A similar effect occurs with an increased $\mathrm{pH}$ due to the application of $\mathrm{CO}_{2}$ in saline (lowest trace in Figure 9D).

\section{Interneurons with rhythmic activity for opo muscles}

Five interneurons of the tg that burst in synchrony with the rhythm of the opos (opo-in) were identified by recording and staining. Their morphology differs considerably; further their physiological influence on the opo muscles ranges from transient influences on the rhythm to resetting the rhythm properties. The basic morphological shapes of these interneurons are (Figure 10A-C): (a) local, with branching restricted to the $\operatorname{tg}$ and connections between ipsilateral areas of adjacent neuromeres, (b) local, with branches crossing the midline, and (c) intersegmental, with axon collaterals entering anterior connectives and preceding ganglia.

Modifying the activity of the interneurons by electrical stimulation influenced the bilateral motor output to the opos in different ways. Three kinds of affects can be characterized: (i) a transient suppression of the bilateral or only the unilateral motor output (Figure 9A, B). The interneuron opo-in 1 has its soma located in the neuromere 9 and branches extensively into all neuromeres but most densely along the median region of the tg. An intersegmental axon collateral ascends in the ipsilateral connective. It exhibits a high tonic spiking activity which can be modulated by irregular bursting. Its effects on the motor output to the opos was most dramatic: when it was released from inhibition the subsequent rebound resulted in 

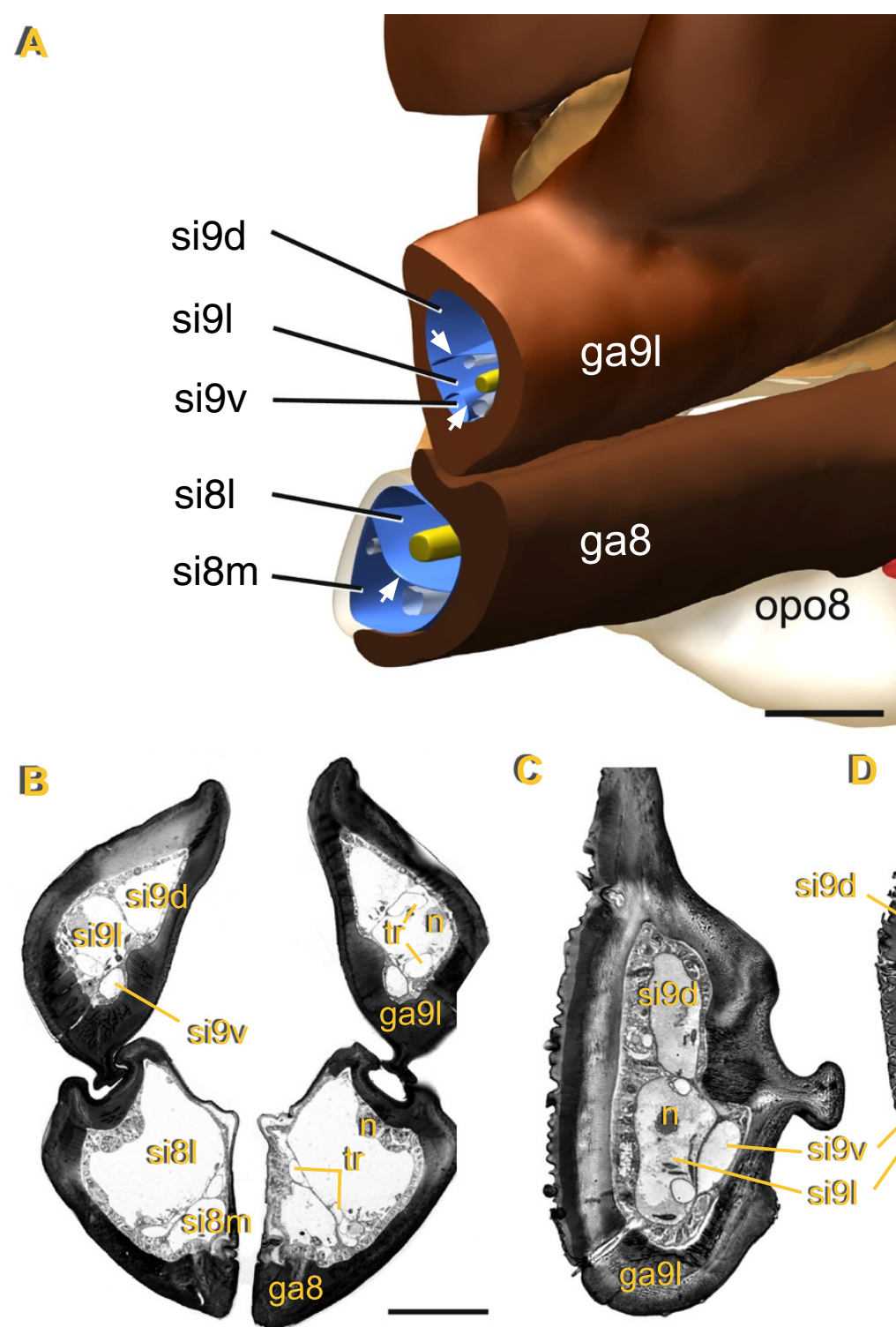

Ф

Figure 5 Morphology and inner organization of the gonapophyses. (A) Reconstruction of right half of ovipositor. Aside from epidermis, gonapophyses contain nerves (yellow), tracheae (gray) and hemolymph; their lumen is divided by thin septa of connective tissue (arrows) into hemolymph sinuses guiding the countercurrent flow; gonapophysis 8 (ga8) with efferent medial sinus (si8m) and afferent lateral sinus (si8l), gonapophysis 9 (ga9) with efferent lateral sinus (si9l), afferent dorsal sinus (si9d) and afferent ventral sinus (si9v) (B) Cross section through paired ga8 and ga9, same level as in diagram above (n: nerves, tr: tracheae). (C, D) Cross sections through most distal region of right ga9, sigv and si9l in (C) merge into one sinus (D), asterisk indicates hemocyte moving through septum gap into neighboring sinus. Scale bar: (A) $150 \mu$ m, (B) $100 \mu \mathrm{m},(\mathbf{C}, \mathbf{D}) 50 \mu \mathrm{m}$.

intense spiking that inhibits the bilateral motor output to the opos specifically on the side ipsilateral to the soma. Nevertheless, the basic ongoing rhythm for the opos was maintained and not reset by the opo-in1. The interneuron opo-in2 (Figure 10B) has a large soma located in the neuromere 8 , and its neurites extend in the ipsilateral neuromeres 8 and 9 and just one branch into the $7^{\text {th }}$ neuromere. The principal axon crosses to the contralateral side, diverges into a smaller posterior branch and then ascends in the contralateral connective to the anterior abdominal ganglion. The opo-in2 bursts in synchrony with the ipsilateral opo motoneurons and when it is hyperpolarized, the ipsilateral opo motoneuron activity is inhibited. That may also slightly affect the basic opo rhythm. (ii) a resetting of the basic rhythm that is achieved by neurons which may be intrinsic to the cpg (published preliminarily as Figure $2 \mathrm{E}$ in [18]). The opo-in3, with a dorsal soma located in the $8^{\text {th }}$ neuromere, extends only in the ipsilateral neuromeres 7 and 8 . It bursts rhythmically in synchrony with the ipsilateral opo 


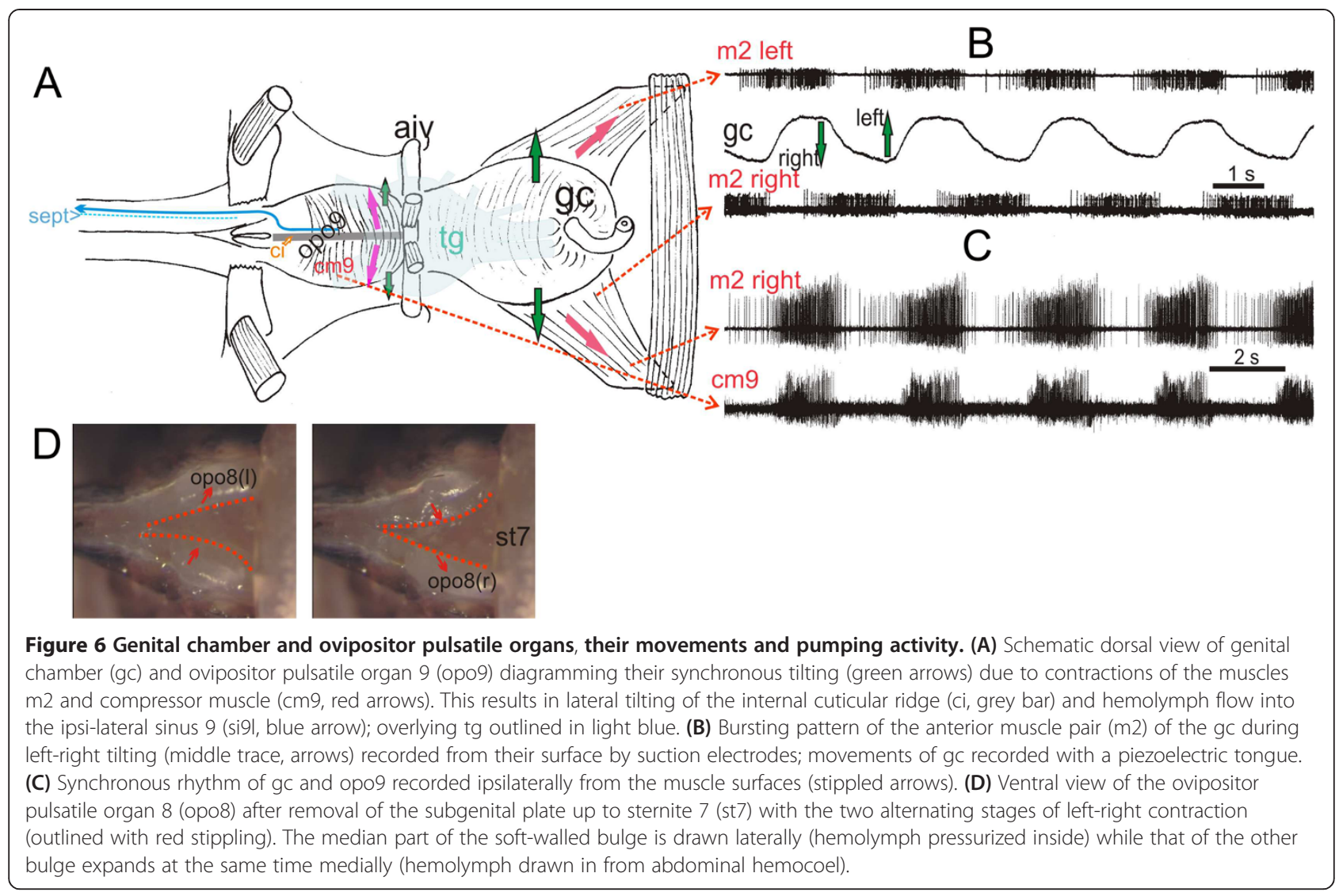

motoneurons. When this rhythm in opo-in3 is abolished by hyperpolarization, the bursting frequency of contralateral opo motoneurons is reduced. Rebounds from inhibition reset the whole opo rhythm starting with ipsilateral excitation and contralateral inhibition. Another interneuron (opo-in4, as APOV-IN4 in [18]) extends ipsilaterally from a particularily posterior and median soma into the $9^{\text {th }}, 8^{\text {th }}$ and $7^{\text {th }}$ neuromere with some smaller branches crossing over the midline. It bursts in synchrony with the contralateral motoneurons of the opos and has the strongest driving and resetting properties for the opo rhythm. Depolarizations of the opo-in4 cause immediate rhythm reset which inhibits the ipsilateral motoneurons and excites the contralateral motoneurons. (iii) an unaltered rhythm by current injection which is observable in the rhythmically active opo-in5 (Figure 10C). This local interneuron connects bilaterally the $8^{\text {th }}$ and $9^{\text {th }}$ neuromeres with widespread branches. Its activity pattern corresponds with the motor bursts that move the opo and gc muscles ipsilateral to the soma. The motor output is not altered dramatically when this neuron is de- or hyperpolarized.

\section{Discussion}

In the accessory circulatory organs of insects one can distinguish between the pulsatile apparatus and the hemolymph guiding structures which provide for circulation throughout the appendage. In part one of the discussion, we address these two construction elements in the cricket ovipositor circulatory organs with respect to their structure and functional mechanisms and compare them with other accessory pulsatile organs [1-3]. The second part of the discussion is dedicated to the neuroanatomical results and the physiological control of the opos.

\section{Functional morphology and pumping mechanism The pulsatile part of the opos}

The pulsatile part of the opos consists of a pump chamber at the base of each valve (functional scheme Figure 11). The organs of the right and left valve of the same segment have a mirrored but otherwise identical anatomy. The organs of the valves of the $8^{\text {th }}$ and $9^{\text {th }}$ segment function according to the same principle, but have a slightly different anatomy. While the pumps at the base of each ga 8 are formed by bulges of flexible cuticle which are compressed by an internal muscle, the pump chambers of the $9^{\text {th }}$ segment are compressed by contractions of an overlying external muscle. Since the compressor muscles of both organs have comparable attachments sites, i.e. laterally at the cs and medially at the flexible cuticle of the base of the ga, they are considered to be 


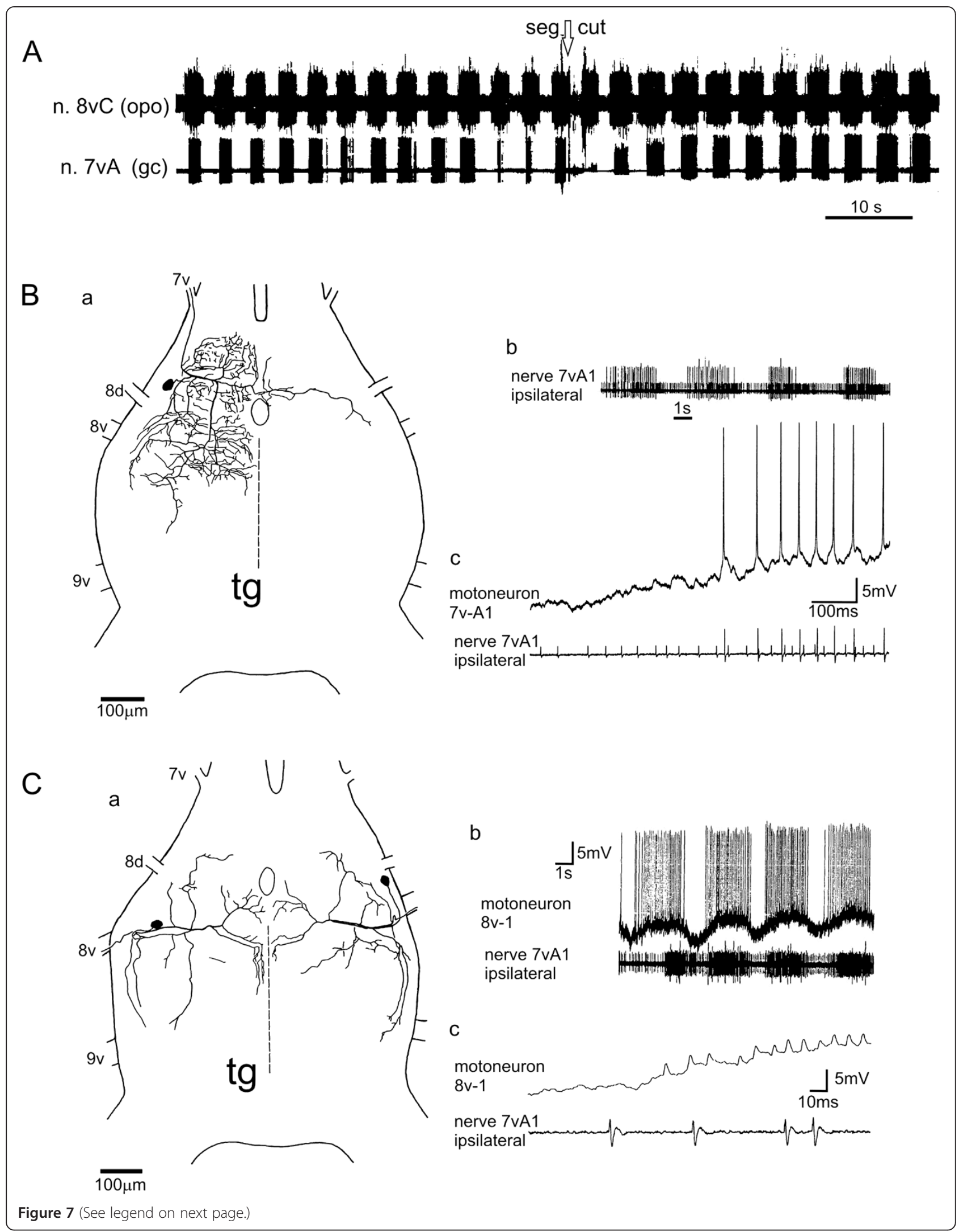


(See figure on previous page.)

Figure 7 Morphology and activity of motoneurons that drive the ovipositor pulsatile organs. (A) Recording from the efferent nerves (suction electrodes) to a opo8 (upper trace, $\mathrm{n} .8 \mathrm{vC}$ ) and to the ipsilateral genital chamber muscle (lower trace, n.7vA, gc) before and after disconnection from cns by severing both connectives (seg cut, arrow) posterior to the subesophageal ganglion. The opo 8 and gc rhythms persist almost undisturbed. (B) a. Branching of one motoneuron for genital chamber movement (7vA-1) in the terminal ganglion (tg, dorsal view) and b. its rhythmic activity along with the second motoneuron to $\mathrm{M} 2$ and, c. onset of a burst due to summation of postynaptic potentials elicited by premotor interneurons. (C) a. Branching types of two icM8 motoneurons in the terminal ganglion (tg, bilateral, dorsal view) which supply the opo8. b. Rhythmic activity of motoneuron $8 v-1$. Summation of excitatory postsynaptic potentials originating from premotor interneurons are the basis for bursting activity of the motoneuron. Lower trace shows synchronous activities of the ipsilateral genital chamber motoneurons. c. Expanded view of the onset of a burst.

serial homologues. Both pumping chambers, as well as the compressor muscles, have not been described in previous anatomical descriptions of the abdominal apex of gryllids (e.g. [19]). The four pumps work continuously whereby the right and left organs of one segment are compressed in alternation. The pumping activities of the $8^{\text {th }}$ and $9^{\text {th }}$ segment are synchronized.

Compared to other accessory pulsatile organs, certain similarities can be found between the functional morphology of the opos and the cercus-hearts in Plecoptera [25]. However, while the cercus-hearts in Plecoptera suck hemolymph out from the cerci into the abdominal cavity, the opos force hemolymph into the valves. Accessory pulsatile organs which likewise force hemolymph into the appendages are the various antenna-hearts; however, they strongly differ in functional morphology and use vessels as hemolymph guiding structures $[11,26,27]$.

\section{Circulation within the valves and tracheal ventilation}

The systolic compression of the pumping chambers force hemolymph distally into the efferent sinus of the valves. The presence of non-return valves could neither be demonstrated in any ga nor at their bases. Probably backflow is reduced by the narrowing of the proximal bases of the pumping chambers during compression. The hemolymph guiding structures are thin septa of connective tissue which extend the whole length of the ovipositor valves up to their apices. There the septa are perforated enabling the passage of hemolymph into the afferent sinuses. Curiously, only one afferent sinus is present in the ga8, while there are two in the ga9. The diameter of the efferent sinuses is much larger in the opo region than that of the afferent sinuses, which may contribute to slowing any backflow when the pump pressure decreases during diastole.

The hemolymph guiding structures in long abdominal appendages of insects are generally vessels [28]. Longitudinal septa which guide the countercurrent hemolymph flow as in the ovipositor valves have been reported from the thoracic legs, the maxillary and labial palps of many insects [3], and the cerci of the cockroach [4]. While in the legs of many Heteroptera, a rhythmically contracting muscle associated with the septum effectuates a countercurrent circulation within the limb $[29,30]$, in most other insects it is not yet fully understood how the observed countercurrent flows are generated $[2,3]$. In some appendages without specific muscular pumps, the breathing-related collapse and expansion of tracheae

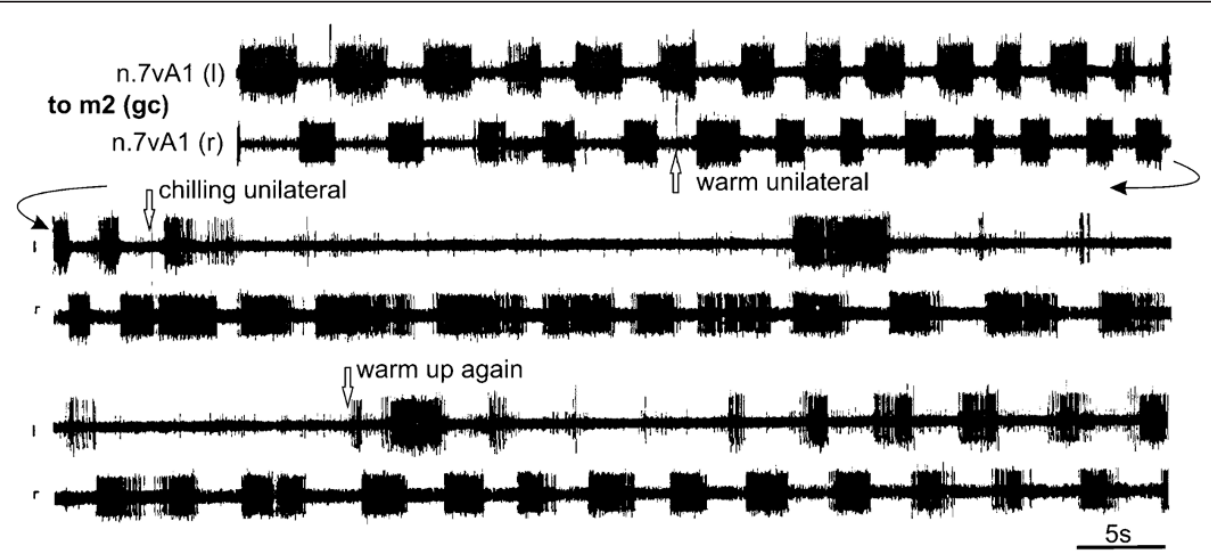

Figure 8 Influences on the rhythmic bilateral genital chamber movements by unilateral warming and cooling. Continuous recording of the efferent output to the genital chamber tilting muscles $\mathrm{m} 2(\mathrm{n} .7 \mathrm{vA} 1 \mathrm{l} / \mathrm{r})$. The left-right rhythm accelerates slightly due to unilateral warming of the terminal ganglion (arrow). Cooling the ganglion with a probe on the other side (arrow, chilling unilateral) abolishes the ipsilateral rhythm and slows the antagonist. Recovery to room temperature $\left(22^{\circ} \mathrm{C}\right.$, arrow) occurs after $60 \mathrm{~s}$. 


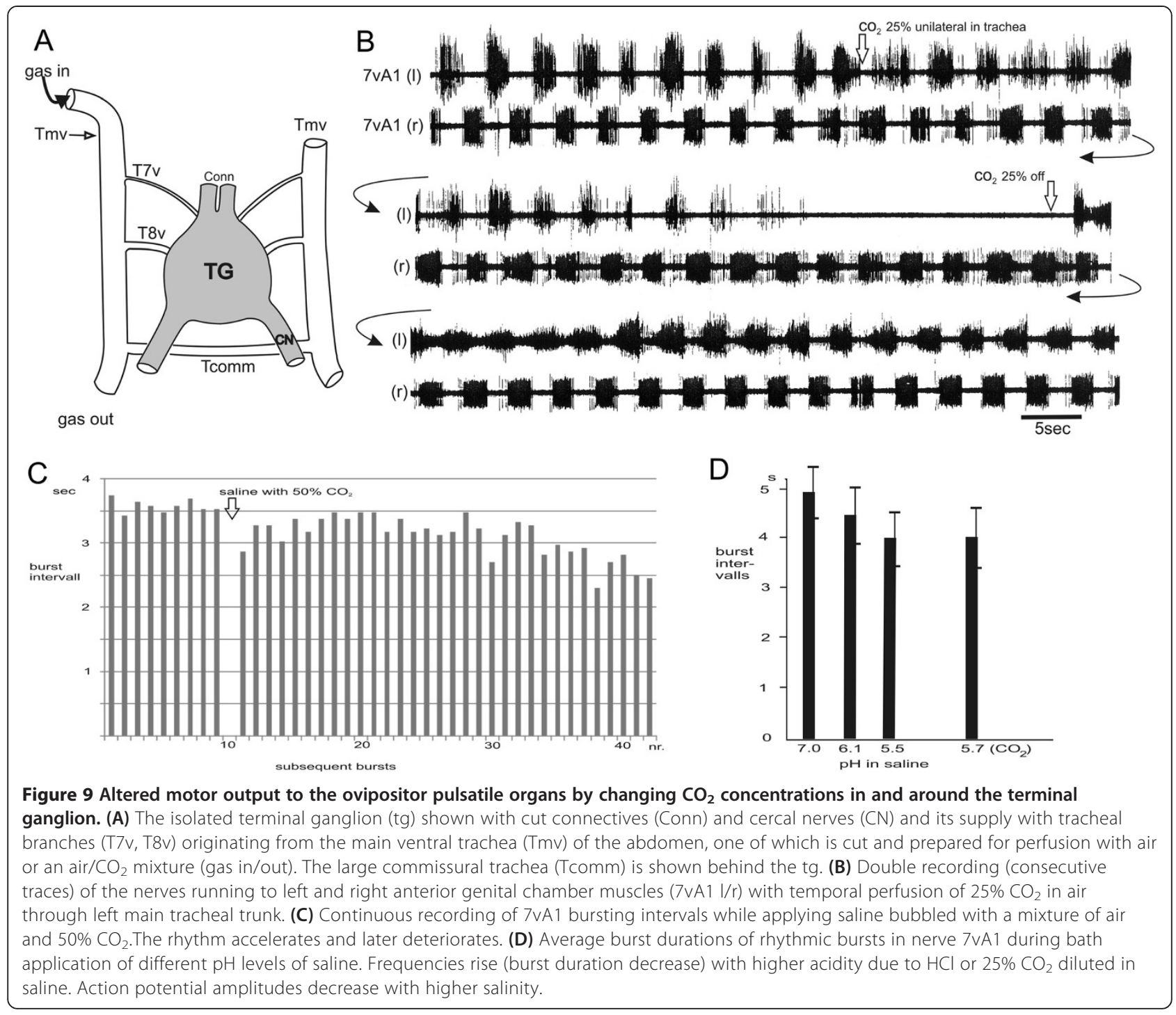

and tracheal sacks cause volume changes that induce hemolymph propagation within the appendage $[5,6]$.

In the cricket ovipositor, the rhythm of the opos is completely independent of ventilatory movements and abdominal compressions. In contrast, in vivo observations show that the pulsed hemolymph flow caused by the rhythmic pumping of the opo results in simultaneous collapses of the widened bases of the tracheae within the ovipositor valves. This clearly must enhance the convection of the tracheal gas and thereby the opos also contribute to the $\mathrm{O}_{2}-\mathrm{CO}_{2}$ gas exchange. A similar relationship between circulation and respiration was also found between the wing circulatory organs and the tracheal tubes in the wing veins [31].

\section{Simultaneous genital chamber movements}

In synchrony with the rhythm of the opos, the apex of the gc moves laterally. We conclude that hemolymph is thereby pressed from the abdomen into the lateral space anterior to the ovipositor base assisting the hemolymph flow toward the ipsilateral ga. Furthermore, the lateral gc movements are probably necessary for hemolymph supply of the entire genitalic region and the abdominal apex since the dorsal heart tube permanently sucks hemolymph away from this region. The gc muscles (m2) always contract in synchrony with the ipsilateral opo8/9. If they contribute to other behavior, e.g. egg laying [32], they can halt or remain in tonic contraction for short periods; the opo8/9 muscles however continue their rhythm in these cases.

\section{Neuroanatomy and physiological control}

In the fused tg both motoneurons and interneurons of the opos tend to extend over several neuromeres. This morphological feature may functionally ease the intersegmental communication between sensory and motor 

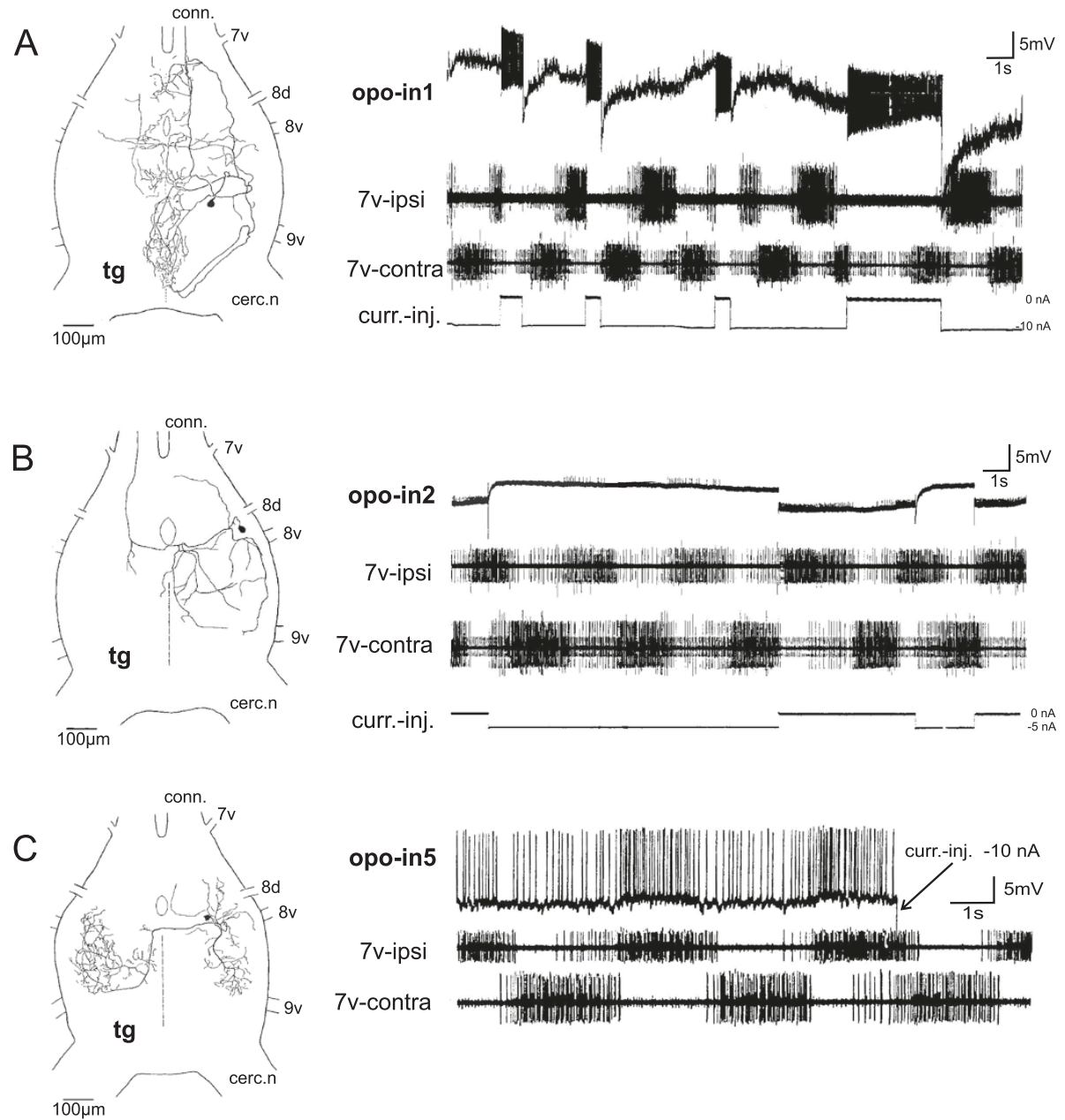

Figure 10 Morphology of terminal ganglion interneurons and their influence on the ovipositor pulsatile organ and genital chamber rhythm. Major nerve roots ( $7 \mathrm{v}-9 \mathrm{~V}$, cerc. n) from neuromeres 7 to 9 are indicated on the terminal ganglion (tg). (A) The opo-in1 is a widespread bilateral interneuron with ipsilateral soma and ascending axon collateral showing little rhythm related activity. Intracellular current injection (curr.inj., lowest trace) elicits an inhibitory effect on the rhythmic output on both sides that is stronger on the ipsilateral motor output to the genital chamber muscles. (B) The opo-in2 is a widespread bilateral interneuron extending over three neuromeres (7-9) with opo motor output, a soma in the neuromere 8 and a contralateral ascending axon collateral. Its own rhythmic activity is enhanced when it is depolarized (bridge balance inverted, current injection in lowest trace) and the efferents to the genital chamber muscles are enhanced ipsilaterally and inhibited contralaterally. (C) The opo-in5 is a bilaterally branching interneuron with extensive branching in neuromeres 8 and 9 . The interneuron bursts in synchrony with the ipsilateral motoneurons but depolarization causes no major changes in rhythm or intensity of motor output.

activity of the adjacent segments, specifically between the rhythmic neurons influencing the pump muscles of the different opos that originate in different neuromeres. Generally, it is rare in insects that the motoneurons innervating non-tergal muscles, such as in the opos, extend with their branches into two or more neighboring ganglia or neuromeres [33]. Basically, interneurons could achieve motoneuron coordination alone when they branch into several neuromeres.

\section{Influences on the coordination of the opo rhythm}

All contractions of opo muscles are coordinated by neuronal control from a common $\mathrm{cpg}$ in the tg. The extent of this neuronal network remains unknown but operates continuously and stably when the ganglion is not addressed by descending neuronal commands.

Higher-order descending interneurons are known to originate in the cricket cns in the subesophageal ganglion serving for the control of respiration [34] and oviposition [35]. Influences on the opo rhythms are evident during strong ventilation or the oviposition procedure when an egg enters the gc and the bilateral muscle pair $\mathrm{m} 2$ contracts synchronously [32]. Comparable systems with autonomous and spontaneous neuronal rhythms are known from other isolated insect ganglia which coordinate, e.g. locust respiration [18,36], cricket oviposition [35], and feeding 


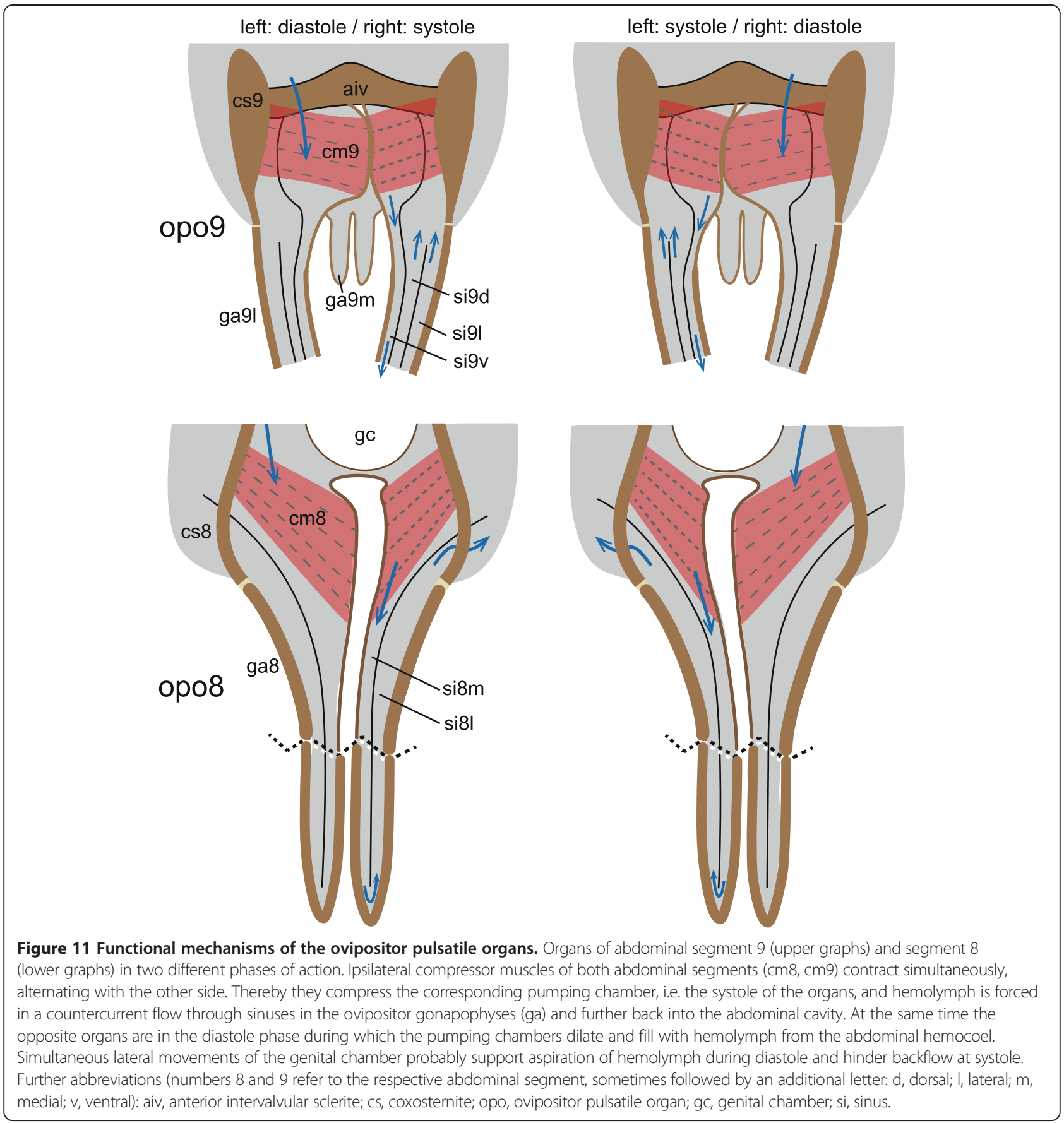

patterns of Drosophila larvae [37]. The autonomous cpg rhythms of these systems appear more "natural" than those which require pharmacological or permanent sensory stimulation such as insect walking [38,39], flying [40], and feeding [41,42].

The autonomous and spontaneous cpg for the opos in the cricket $\operatorname{tg}$ can be modulated by the following non-neural factors: (i) temperature changes that induce activity changes of the cpg and (ii) lowered $\mathrm{pH}$ in the bathing fluid provided by an increased $\mathrm{pCO}_{2}$ causing rhythm acceleration. In contrast, when higher levels of $\mathrm{pCO}_{2}$ are introduced into the tg via its tracheal supply, the effect is not rhythm acceleration but rather that of an anesthetic. These contrasting $\mathrm{CO}_{2}$-effects may reach the cpg in the neuropil by different mechanisms. The rapid effect of $\mathrm{pH}$ changes in the bathing fluid may be transferred inward by the glial cells which are interconnected with numerous gap junctions [43]. They may transmit the effect to the cpg neurons for the opos. As an alternative explanation, specific sensory neurons with 
endings near the surface of a ganglion may monitor $\mathrm{pH}$ changes and influence the neurons inside the ganglion but sensors of this type are so far not known from any insect cns.

The contrasting (non-pH-like) effect of $\mathrm{CO}_{2}$ after intra-tracheal infusion inhibits the rhythmic motor output of only the ipsilateral hemiganglion of the tg. This speaks against a $\mathrm{pH}$-effect via the ganglion surface and agrees with the notion that there is no tracheal junction over the midline to the contralateral side of the tg [44]. Apparently the gaseous intratracheal $\mathrm{CO}_{2}$ has a low effect on the $\mathrm{pH}$ levels in the environment of the cpg neurons of the tg. That seems to indicate a neuronal tolerance to self-produced metabolic $\mathrm{CO}_{2}$ in the cns, as was found for single neurons of crickets [45].

The hemolymph that returns from the ovipositor partly overflows the tg with metabolically loaded and more acidic hemolymph caused by a high metabolic rate of the many cuticular sensilla located on the surface of the ovipositor [46]. That may contribute to the regulation of the cpg rhythm as indicated by experimental superfusion of the isolated tg. In this way metabolic requirements may indirectly control the velocity of the hemolymph flow through the ovipositor valves.

\section{Coupling of the left-right opo rhythm}

Unilateral changes of external influences on the tg, such as cooling, and unilateral application of $\mathrm{CO}_{2}$, affect the rhythmic output mainly on the ipsilateral side, at least, for the first minutes of application (Figure 9); the rhythm on the other side remains nearly unchanged. This strong ipsilateral suppression of the motor, and possibly also of premotor neurons, raises the question whether the total ipsilateral cpg is affected. That leads one to assume that the cpg for the opos consists of two (left and right) half centers producing their own - but normally coupled rhythms.

\section{Interneurons and the cpg}

All interneurons exhibiting the rhythm of the opos could belong to the cpg itself or are influenced by it. They extend over, at least, two or more neuromeres of the tg. A similarly extensive wiring is required to connect the cpg to the different motoneurons of the segmental neuromeres 7-9 which has efferents to the opos and gc. Yet the exact location of the rhythm-generating neuronal network and the extent of the essential network remain unclear. The "core" of the cpg may be located in the neuromere 8 where all the motoneurons for the rhythmic muscles have branches. At the level of interneurons, only one potentially rhythm resetting interneuron (opo-in3) was found that branches unilaterally in neuromeres 8 and 9, whereas the opo-in4 reaches all neuromeres mainly on one side and the contralateral neuromere 8 [18]. In contrast, the opo-in5 exhibits a morphology that appears well suited for a left-right coordination of all opo-rhythms. However, the physiology of this interneuron, with its ideal left-right connection and rich bilateral arborizations in the neuromeres 8 and 9 , is not sufficiently elaborated to substantiate the proposed function.

\section{Conclusions}

Most arthropods have a complex vascular system in which the limbs are supplied with hemolymph by arteries. In insects, this system is greatly reduced and a ventral longitudinal vessel from which such arteries could emanate is lacking [47]. Their thoracic limbs are supplied by sinuses delimited by thin septa of connective tissue which are perforated in the tip region of the appendage enabling a countercurrent hemolymph flow [3]. A comparable condition can also be found in the gonapophyseal appendages in Acheta. However, while in most thoracic limbs and cerci it is unclear how the hemolymph flow is generated, a pumping apparatus exists for each of the ovipositor valves. These organs represent evolutionary novelties having a functional morphology which has not been reported from any other auxiliary heart in insects [2]. The origin of the associated pumping muscle must remain unclear since no unambiguous homologization with any of the serial homologues of the abdominal musculature is possible.

With respect to physiological control, it must be emphasized that the neurogenic automatism of the opo is unique among insects. All other known circulatory organs are based on a myogenic automatism which may be neuronally or hormonally modulated [6-10]. The great autonomy of opo rhythm generation is surprising. The only noticeable influence on the cpg interneurons is - apart from general temperature effects and inhibitory cns commands - the $\mathrm{pH}$ of the fluid surrounding the tg. This may be linked with the metabolic requirements of the numerous sensilla which are located especially at the ovipositor apex. An additional task of the opos may be the convection of the extensive tracheal system within the ovipositor valves.

From an evolutionary point of view it will be a rewarding task to investigate if corresponding pump organs are associated with the ovipositors in other insects. Future research in this direction could reveal remarkable insights to the evolution of the female ovipositor in insects, a classical topic of comparative morphology in these animals $[19,21,22,48-50]$.

\section{Material and methods Animals}

Females of Acheta domesticus used in this study originated from breeding stocks in our laboratories. For immobilization the specimens were cooled to $0-4^{\circ} \mathrm{C}$ 
previous to and during preparations. All experiments were carried out respecting the relevant ethical guidelines for experimentation with live animals.

\section{Observation of the pumping organs in vivo}

The speed and direction of hemolymph flow inside the ovipositor valves is readily recognizable through the transparent regions of the ovipositor cuticle via movement of the hemocytes. Experiments with introducing various vital stains into the hemolymph failed due to immediate clotting that slowed or stopped fluid propagation in the small sinuses of the ovipositor. Observations were made with incident or translucent light under a stereomicroscope. In addition, the pumping action of the opos was videorecorded (camera: Kappa $\mathrm{C} 15$ ) in intact animals (in a small glass chamber from below), as well as from prepared specimens (ventral side up). The range of peak velocities during pumping strokes was calculated $(n=8$ preparations) from tracking individual large hemocytes frame by frame in high-speed video sequences (300 fps, Casio Exilim F1) recorded through a dissection microscope in translucent light.

Correlation of the hemolymph pulses in the ovipositor valves to the pumping activity of the opo8 was studied from the ventral side after removal of the subgenital plate that covers the ovipositor base ventrally. The pumping movements of the opo9 system were observed dorsally in semi-intact preparations after removal of overlying muscles and other tissue.

Experimentally induced influences on the opo/gc rhythm were measured in 5-8 animals per parameter, relating the undisturbed burst frequency of the individual preparation with the altered frequency after introducing an influence to the same preparation.

\section{Morphological methods}

Chemical fixation: freshly cut last abdominal segments of female crickets were fixed in alcoholic Bouin ("Dubosq-Brasil" mixture) and subsequently washed in ethanol.

Histological sections: the fixed specimens were embedded after dehydration with acetone in low viscosity resin (Agar Scientific). Serial semithin sections ( $1 \mu \mathrm{m}$ thickness) were cut with a diamond knife on an ultramicrotome and stained with a mixture of $1 \%$ azure II and $1 \%$ methylene blue in a $1 \%$ aqueous borax solution for approximately $40 \mathrm{~s}$ at $80^{\circ} \mathrm{C}$.

MicroCT: a female abdomen fixed in alcoholic Bouin was stained in a solution of $1 \%$ iodine in $96 \%$ ethanol overnight. After this treatment it was imaged with an Xradia MicroXCT x-ray microtomography system (University of Vienna, Department of Theoretical Biology) with a tungsten source at $60 \mathrm{kVp}$ and $66 \mu \mathrm{A}$.
$3 \mathrm{D}$ reconstruction and visualization: the software Amira 5.4.2 was used for $3 \mathrm{D}$ reconstruction of the microCT dataset. Blender (www.blender.org) was used to postprocess the meshes exported from Amira and to remodel certain parts using the Amira data as a guide. Images of semi-thin sections were postprocessed with Fiji (www. fiji.sc) using the CLAHE plugin to enhance contrast.

\section{Recording from nerves and muscles}

To make preparations of the dorsal side, the median part of the tergites, the gut and the ovaries were removed carefully. That gave access to the tg, peripheral nerves, several muscles of the opos and the gc. The easiest access for recording is to the opo muscles (m2) and its motor nerve $7 \mathrm{vA}$ whose bursting activities are always in synchrony with the ipsilateral opo muscles $\mathrm{cm} 8 / 9$. The internal organs were flushed regularly with saline [51]. Care was taken not to block the abdominal spiracles by saline from outside. Extracellular recording was performed with suction electrodes on cut nerve stumps, laterally on intact nerves, or by gently sucking the surface of active muscles near their attachments where movement amplitudes of the fibers were low. The time intervals from the start of a burst to the next burst (myogram or nerve recording) were measured continuously for several hours in more than 25 specimens. In none of these or any of the other 250-300 experiments we found rhythms below $0.2 \mathrm{~Hz}$ or above $0.5 \mathrm{~Hz}$ at room temperature.

Intracellular recording required a supporting silver platform for the tg. The electrodes for intracellular recording were made of borosilicate glass with 50-80 $\mathrm{M} \Omega$ tip resistances and had their shaft filled with $1 \mathrm{M}$ $\mathrm{LiCl}$ while their tip contained about 1-2\% Lucifer yellow in $\mathrm{LiCl}$ for iontophoretic staining. Intracellular recording focused on rhythmically active or rhythm-influencing interneurons and motoneurons; the data were stored on magnetic tape (Racal Store 7) or on a PC after digitalization (Datapac K2).

Temperature application ( $\mathrm{n}=6$ preparations): Short metal studs connected to a regulated Peltier element (Peltron, Nürnberg) were brought close to the tg laterally with temperatures of either $0^{\circ}$ or $25^{\circ}$ Celsius.

Superfusion and infusion of gas mixtures: The different gas mixtures were mixed before application in a gas syringe and each type of experiment was repeated 5 to 8 times.

\section{Terminology}

A confusing multitude of synonyms exist for the ovipositor valves and linked structures (see Scudder [21]). For reasons of comprehensibility we use "gonapophysis" as a descriptive term to refer to all three valves forming the ovipositor shaft in Acheta without implying homology. The numbering of 
some muscles was taken from the descriptions for Gryllus assimilis by Snodgrass [19]. The nerve roots of the terminal ganglion were named according to the abdominal segment that they supply, e.g. 8d supplying the dorsal region of the $8^{\text {th }}$ segment and $8 \mathrm{v}$ for the ventral region.

\section{Additional file}

Additional file 1: Video. The clip is divided into three sections. (1) Bases of the gonapophyses 8 are shown in ventral view displaying the left-right alternating pumping of the ovipositor pulsatile organs 8 (subgenital plate removed). The compression of one bulge supports the dilation of the other bulge as it fills up with hemolymph drawn from the abdominal body cavity. Note the alternating lateral shift of the flat cuticular area between the two bulges which narrows the hemolymph connection to the abdominal hemocoel and which probably prevents backflow. (2a) Countercurrent flow of hemocytes in the efferent ga8m (flow direction: left) and the afferent ga8l (flow direction: right), ventral view. The flow pulses alternate between the right (upper one in the video) and left gonapophysis 8 (2b) Collapsing tracheae in the proximal gonapophysis 8 , ventral view. (3) Dorsal view of the muscles of the ovipositor pulsatile organ 9 , which are active in the alternating pumping movements visible below the terminal ganglion (overexposed; cercal nerves cut). The whitish processes at the end of ovipositor pulsatile organ 9 are the median gonapophyses 9 .

\section{Abbreviations \\ Appended numbers 8 and 9 refer to the concerned abdominal segment; $\mathrm{cm}$ : Compressor muscle; cns: Central nervous system; cpg: Central pattern generator; cs: Coxosternite; ga: Gonapophysis; gc: Genital chamber; opo: Ovipositor pulsatile organ; opo-in: Ovipositor pulsatile organ interneuron; tg: Terminal ganglion.}

\section{Competing interests}

The authors declare that they have no competing interests.

\section{Authors' contributions}

$\mathrm{RH}$ and GP discovered the opos in Acheta independently and later combined their efforts. The morphological investigations were carried out by $A B, R H$, and $G P$, the neuroanatomical and physiological analysis by $R H$ and MF. The text was written by RH, GP and AB. All authors read and approved the final version of the manuscript.

\section{Acknowledgements}

The authors thank Julia Bauder for her careful technical assistance and Christina Heindl for taking photographs. The microCT scan was performed at the Department of Theoretical Biology of the University Vienna and we acknowledge Brian Metscher for his help. Many thanks also to John Plant for improving the English. The study was financially supported by the Austrian science fund FWF project 23251-B17.

\section{Author details}

'Department of Neurobiology, JFB-Institute for Zoology, University of Göttingen, Berliner Straße 28, 37073 Göttingen, Germany. ${ }^{2}$ Department of Integrative Zoology, University of Vienna, Althanstraße 14, 1090 Vienna, Austria.

Received: 27 March 2014 Accepted: 31 May 2014

Published: 8 June 2014

\section{References}

1. Pass G: Accessory pulsatile organs. In Microscopic Anatomy of Invertebrates, Volume 11B. Edited by Harrison F, Locke M. New York: Wiley-Liss: 1998:621-640.

2. Pass G: Accessory pulsatile organs: evolutionary innovations in insects. Annu Rev Entomol 2000, 45:495-518.

3. Pass G, Gereben-Krenn B-A, Merl M, Plant J, Szucsich NU, Tögel M: Phylogenetic relationships of the orders of Hexapoda: contributions from the circulatory organs for a morphological data matrix. Arthr Syst Phyl 2006, 64:165-203.

4. Murray JA: Morphology of the cercus in Blattella germanica (Blattaria: Pseudomopinae). Ann Entomol Soc Am 1967, 60:10-16.

5. Wasserthal LT: The open hemolymph system of Holometabola and its relation to the tracheal space. In Microscopic Anatomy of Invertebrates, Volume 11B. Edited by Harrison F, Locke M. New York: Wiley-Liss; 1998:583-620

6. Hustert R: Accessory hemolymph pump in the middle legs of locusts. Int J Insect Morphol \& Embryol 1999, 28:91-96.

7. Miller TA: Structure and physiology of the circulatory system. In Comprehensive insect physiology, biochemistry and pharmacology, Volume 3. Edited by Kerkut GA, Gilbert LI. Oxford: Pergamon Press; 1985:289-353.

8. Miller TA: Control of circulation in insects. Gen Pharmacol 1997, 29:23-38.

9. Hertel W, Pass G: An evolutionary treatment of the morphology and physiology of circulatory organs in insects. Comp Biochem and Physiol A 2002, 133:555-575.

10. Miller T, Pass G: Circulatory system. In Encyclopedia of insects. 2nd edition. Edited by Resh VH, Cardé RT. Burlington: Elsevier; 2009:169-173.

11. Pass $\mathrm{G}:$ Gross and fine structure of the antennal circulatory organ in cockroaches (Blattodea, Insecta). J Morphol 1985, 185:255-268.

12. Hertel W, Pass G, Penzlin H: Electrophysiological investigation of the antennal heart of Periplaneta americana and its reactions to proctolin. $J$ Insect Physiol 1985, 31:563-572.

13. Pass G, Agricola H, Birkenbeil H, Penzlin H: Morphology of neurones associated with the antennal heart of Periplaneta americana (Blattodea, Insecta). Cell Tissue Res 1988, 253:319-326.

14. Pass G, Sperk G, Agricola H, Baumann E, Penzlin H: Octopamine in a neurohaemal area within the antennal heart of the American cockroach. J Exp Biol 1988, 135:495-498.

15. Hertel W, Richter M: Contributions to physiology of the antenna-heart in Periplaneta americana (L.) (Blattodea: Blattidae). J Insect Physiol 1997, 43:1015-1021.

16. Hertel W, Neupert S, Eckert M: Proctolin in the antennal circulatory system of lower Neoptera: a comparative pharmacological and immunohistochemical study. Physiol Entomol 2012, 37:160-170

17. Frisch $M$, Hustert $R: A$ central pattern generator in the terminal ganglion of a cricket. In Gene - Brain - Behaviour. Edited by Elsner N, Heisenberg M. Stuttgart: Thieme Verlag; 1993:80.

18. Hustert R, Mashaly AM: Spontaneous behavioral rhythms in the isolated CNS of insects - Presenting new model systems. J Physiol-Paris 2013, 107:147-151.

19. Snodgrass RE: Morphology of the insect abdomen. II. The genital ducts and the ovipositor. Smith Misc Coll 1933, 89:1-147.

20. Snodgrass RE: Principles of Insect Morphology. New York: McGraw-Hill; 1935.

21. Scudder GGE: The comparative morphology of the insect ovipositor. Trans Roy Entomol Soc London 1961, 113:25-40.

22. Klass KD, Ulbricht J: The female genitalic region and gonoducts of Embioptera (Insecta), with general discussions on female genitalia in insects. Org Div \& Evol 2009, 9:115-154.

23. Sakai $\mathrm{M}$, Kumashiro $\mathrm{M}$ : Copulation in the cricket is performed by chain reaction. Zool Sci 2004, 21:705-718.

24. Hustert R: Rhythmogenesis in a segmental neural network for respiration in an insect. In Rhythmogenesis in neurons and networks. Edited by Elsner $\mathrm{N}$, Richter D. Stuttgart: Thieme Verlag; 1992:62.

25. Pass G: The "cercus heart" in stoneflies - a new type of accessory circulatory organ in insects. Naturwiss 1987, 74:440-441.

26. Pass G: Functional morphology and evolutionary apsects of unusual antennal circulatory organs in Labidura riparia Pallas (Labiduridae), Forficula auricularia L. and Chelidurella acanthopygia Géné (Forficulidae) (Dermaptera: Insecta). Int J Insect Morph Embryol 1988, 17:103-112.

27. Pass G: Antennal circulatory organs in Onychophora, Myriapoda and Hexapoda: functional morphology and evolution. Zoomorphology 1991, 110:145-164.

28. Gereben-Krenn BA, Pass G: Circulatory organs of abdominal appendages in primitive insects (Hexapoda: Archaeognatha, Zygentoma and Ephemeroptera). Acta Zool-Stockholm 2000, 81:285-292.

29. Debasieux P: Organes pulsatiles des tibias de Notonectes. Ann Soc Sci Brux B 1936, 56:77-87. 
30. Hantschk AM: Functional morphology of accessory circulatory organs in the legs of Hemiptera. Int J Insect Morphol \& Embryol 1991, 6:259-273.

31. Wasserthal LT: Oscillating haemolymph 'circulation' and discontinuous tracheal ventilation in the giant silk moth Attacus atlas L. J Comp Physio/ 1980, 145:1-15.

32. Sugawara T, Loher W: Oviposition behavior of the cricket Teleogryllus commodus: Observation of external and internal events. J Insect Physiol 1986, 32:179-188.

33. Steffens GR, Kutsch W: Homonomies within the ventral muscle system and the associated motoneurons in the locust, Schistocerca gregaria (Insecta, Caelifera). Zoomorpology 1995, 115:133-155.

34. Otto D, Janiszewsky J: Interneurones originating in the suboesophageal ganglion that control ventilation in two cricket species: Effects of the interneurones (SD-AE neurones) on the motor output. J Insect Physiol 1989, 35:483-491.

35. Ogawa H, Kagaya K, Saito M, Yamaguchi T: Neural mechanism for generating and switching motor patterns of rhythmic movements of ovipositor valves in the cricket. J Insect Physiol 2011, 57:326-338.

36. Bustami HP, Hustert R: Typical ventilatory pattern of the intact locust is produced by the isolated CNS. J Insect Physiol 2000, 46:1285-1293.

37. Schoofs A, Niederegger S, Spieß R: From behavior to fictive feeding: Anatomy, innervation and activation pattern of pharyngeal muscles of Calliphora vicina 3rd instar larvae. J Insect Physiol 2009, 55:218-230.

38. Ryckebusch S, Laurent G: Rhythmic patterns evoked in locust leg motor neurons by the muscarinic agonist pilocarpine. J Neurophysiol 1993, 69:1583-1595.

39. Johnston RM, Levine RB: Crawling motor patterns in the isolated larval nerve cord of Manduca sexta induced by pilocarpine. In: Neural Systems and Behaviour. Proceedings of the Fourth International Congress of Neuroethology, Cambridge UK, 3.-8.Sept: Edited by Burrows M, Matheson T, Newland PL, Schuppe H. Stuttgart: Thieme Verlag 1995, 1995:470.

40. Wilson DM: The central nervous control of flight in a locust. J Exp Biol 1961, 38:471-490.

41. Rast GF, Bräunig P: Pilocarpine-induced motor rhythms in the isolated locust suboesophageal ganglion. J Exp Biol 1997, 200:2197-2207.

42. Bowdan E, Wyse GA: Temporally patterned activity recorded from mandibular nerves of the isolated subesophageal ganglion of Manduca. J Insect Physiol 2000, 46:709-719.

43. Swale LS, Lane NJ: Embryonic development of glial cells and their junctions in the locust central nervous system. J Neurosci 1985, 5:117-127.

44. Longley A, Edwards JS: Tracheation of abdominal ganglia and cerci in the house cricket Acheta domesticus (Orthoptera, Gryllidae). J Morphol 1997, 159:233-243.

45. Clark MA, Eaton DC: Effect of $\mathrm{CO}_{2}$ on neurons of the house cricket, Acheta domestica. J Neurobiol 1983, 14:237-250.

46. Markus B: Untersuchungen zur Sensomotorik des Ovipositors bei Grillen. Diplomarbeit, Universität Köln 1985. As cited. In Cricket behaviour and neurobiology. Edited by Huber F, Moore TE, Loher W. Ithaca and London: Comstock/Cornell University Press; 1989:74.

47. Wirkner S, Tögel M, Pass G: The arthropod circulatory system. In Arthropod Biology and Evolution, Molecules, Development, Morphology. Edited by Minelli A, Boxshall G, Fusco G. Berlin, Heidelberg: Springer; 2013:343-391.

48. Crampton GC: The terminal abdominal structures of female insects compared throughout the orders from the standpoint of phylogeny. J New York Entomol Soc 1929, 37:453-496.

49. Rousset $A$ : Squelette et musculature des regions génitals et postgénitales de la femelle de Thermobia domestica (Packard). Comparaison avec la region génital de Nicoletia sp. (Insecta: Apterygota: Lepismatida). Int J Insect Morph \& Embryol 1973, 2:25-85.

50. Klass KD, Matushkina NA: The exoskeleton of the female genitalic region in Petrobiellus takunagae (Insecta: Archaeognatha): Insect-wide terminology, homologies, and functional interpretations. Arthropod Struct Dev 2012, 41:575-591.

51. Eibl E: Morphology of the sense organs in the proximal part of the tibiae of Gryllus campestris L. and Gryllus bimaculatus de Geer (Insecta, Ensifera). Zoomorphology 1978, 89:185-205.

doi:10.1186/1742-9994-11-43

Cite this article as: Hustert et al:: A new kind of auxiliary heart in insects: functional morphology and neuronal control of the accessory pulsatile organs of the cricket ovipositor. Frontiers in Zoology 2014 11:43.

\section{Submit your next manuscript to BioMed Central and take full advantage of:}

- Convenient online submission

- Thorough peer review

- No space constraints or color figure charges

- Immediate publication on acceptance

- Inclusion in PubMed, CAS, Scopus and Google Scholar

- Research which is freely available for redistribution 